\title{
ROBUST PRIOR-BASED DIRECTION OF ARRIVAL ESTIMATION
}

\author{
Petter Wirfält, Magnus Jansson \\ ACCESS Linnaeus Center, Electrical Engineering/Signal Processing lab \\ KTH Royal Institute of Technology, Sweden
}

\begin{abstract}
In certain Direction of Arrival (DOA) scenarios some of the sources are approximately known a priori. It is then desirable to be able to exploit this prior knowledge when estimating the DOAs of the unknown sources. In this paper we modify an estimator utilizing exact angular prior knowledge of some sources such that the estimator is able to exploit prior knowledge with some uncertainty. We derive the corresponding Cramér-Rao lower bound and present numerical results showing that the estimator can benefit from prior information, even when it is inaccurate.
\end{abstract}

Index Terms - Direction of arrival estimation; array signal processing; Bayesian estimation

\section{INTRODUCTION}

Direction of Arrival (DOA) estimation is one of the most widely researched areas in Signal Processing. In certain applications some of the received signals are coming from a known direction. These signals can for example be from an obstacle in the field of view (FOV) of the sensing array, or perhaps from a friendly target whose location is known.

Most DOA algorithms estimate all sources in the received signal. For such algorithms a known source among the unknown will be treated as another parameter to estimate, and as such will (in general) degrade the accuracy of the estimates of the other sources.

Several methods have been proposed to exploit the prior knowledge of DOAs in the array FOV. Some use orthogonal projections which in this context means that the received signal is orthogonally projected onto a subspace which does not contain the known signal direction(s) [1]. Recently, a new method of exploiting such knowledge, denoted PLEDGE, was introduced [2]. Especially in some scenarios, this method gave a dramatic increase in estimation accuracy [3].

However, both the above mentioned methods suffer from that they require the exact angular knowledge of the sources; no uncertainty is accounted for in the prior information. In some scenarios this is valid; the position of the known source is exact. In other scenarios it is more proper to model a known DOA as having a nominal value with some uncertainty. This could for example be the case when a friendly target is reporting its GPS-position; then usually a quantitative measure of the uncertainty is also provided.

In this paper we propose a method to accurately exploit such uncertain known sources. The method requires some nominally known DOAs and a measure of the uncertainty in said DOAs. As will be shown this translates into a method that adaptively values the prior

The research leading to these results has received funding from the European Research Council under the European Community's Seventh Framework Programme (FP7/2007-2013) / ERC grant agreement \#228044 information in proportion to the uncertainty; this allows the method to benefit from prior DOA information, even when that information is not absolutely accurate.

The idea in this paper is to model the uncertainty in the prior as an error in the receiving array. Seen from this perspective, it is possible to utilize results pertaining to methods designed to be robust to array errors [4], [5]. One such estimator, which in addition has the attractive property of being relatively straight-forward to realize for a uniform linear array, is [6], and we apply the general framework in that article to our specific case.

\section{PROBLEM FORMULATION}

We consider the case of $d$ narrow-band, plane waves impinging on an $m$ sensor uniform linear array. Of these $d$ waves, $d_{k}$ are emanating from sources whose positions are known with some uncertainty. We can formulate a model for the received data according to

$$
\mathbf{x}(t)=\mathbf{A}(\boldsymbol{\theta}) \mathbf{s}(t)+\mathbf{n}(t), \quad t=1,2, \ldots, N,
$$

where $\mathbf{A}(\boldsymbol{\theta})$ is the array steering matrix parameterized by the vector of DOAs $\boldsymbol{\theta}=\left[\begin{array}{ll}\boldsymbol{\theta}_{u}^{\mathrm{T}} & \boldsymbol{\theta}_{k}^{\mathrm{T}}\end{array}\right]^{\mathrm{T}}$, where the subscripts $u$ and $k$ denote unknown and known, respectively, and ${ }^{\mathrm{T}}$ is the transpose operation. In addition, $\mathbf{s}(t)$ is the source signal waveforms sampled at time $t$, and $\mathbf{n}(t)$ the sensor noise. The $i$ :th column of $\mathbf{A}$ is defined as

$$
\mathbf{a}\left(\theta_{i}\right)=\left[\begin{array}{llll}
1 & e^{j \phi_{i}} & \ldots & e^{j(m-1) \phi_{i}}
\end{array}\right]^{T}, \quad i=1, \ldots, d,
$$

where

$$
\phi_{i}=-2 \pi \Delta \sin \left(\theta_{i}\right)
$$

and $\Delta$ is the sensor separation in wavelengths. The complexGaussian, zero-mean signal and noise sequences $\mathbf{s}(t)$ and $\mathbf{n}(t)$ are characterized by their second order statistics, which are assumed to be

$$
\begin{aligned}
\mathrm{E}\left[\mathbf{s}(t) \mathbf{s}^{*}(\tau)\right] & =\mathbf{P} \delta(t, \tau), \\
\mathrm{E}\left[\mathbf{n}(t) \mathbf{n}^{*}(\tau)\right] & =\sigma^{2} \mathbf{I}_{m} \delta(t, \tau),
\end{aligned}
$$

where ${ }^{*}$ denotes conjugate transpose and $\mathbf{I}_{m}$ denotes the identity matrix of size $m \times m$. For coherent sources, the rank of $\mathbf{P}$ is less than $d$ and accordingly we let $d^{\prime}=\operatorname{rank}(\mathbf{P})$.

The contribution of this paper is the design of an estimator that can take some uncertainty in the known DOAs into account. Accordingly, we model the known DOAs as Gaussian random variables independent of $\mathbf{s}(t)$ and $\mathbf{n}(t)$ defined by their mean $\mathrm{E}\left(\boldsymbol{\theta}_{k}\right)=\overline{\boldsymbol{\theta}}_{k}$ and their covariance

$$
\mathrm{E}\left[\left(\boldsymbol{\theta}_{k}-\overline{\boldsymbol{\theta}}_{k}\right)\left(\boldsymbol{\theta}_{k}-\overline{\boldsymbol{\theta}}_{k}\right)^{\mathrm{T}}\right]=\boldsymbol{\Theta} .
$$

In (6), $\Theta$ is "small" in the sense that only first order perturbations in $\boldsymbol{\theta}_{k}$ around $\overline{\boldsymbol{\theta}}_{k}$ need to be considered in the ensuing analysis. We 
assume we have access to the prior knowledge of the mean position $\overline{\boldsymbol{\theta}}_{k}$ and the covariance $\boldsymbol{\Theta}$; the idea is that the estimator should in some sense weight the knowledge of the known DOA according to this covariance.

\section{DOA ESTIMATOR}

We begin by looking at a general description of a DOA-estimator; we will then introduce the concept of exact knowledge of some DOAs, and finally modify that estimator such that we can account for the stated uncertainty in the known DOAs. Let

Some additional statistics is needed in order to accomplish this.

$$
\mathrm{E}\left[\mathbf{x}(t) \mathbf{x}^{*}(t)\right] \triangleq \mathbf{R}=\mathbf{A}_{0} \mathbf{P} \mathbf{A}_{0}^{*}+\sigma^{2} \mathbf{I}_{m},
$$

where $\mathbf{A}_{0}=\mathbf{A}\left(\overline{\boldsymbol{\theta}}_{u}, \overline{\boldsymbol{\theta}}_{k}\right)$ and $\overline{\boldsymbol{\theta}}_{u}$ denotes the true positions of the unknowns sources. Performing an eigendecomposition of (7) then gives

$$
\mathbf{R}=\mathbf{E}_{s} \boldsymbol{\Lambda}_{s} \mathbf{E}_{s}^{*}+\mathbf{E}_{n} \boldsymbol{\Lambda}_{n} \mathbf{E}_{n}^{*}
$$

where $\mathbf{E}_{s} \in \mathbb{C}^{m \times d^{\prime}}$ contains the $d^{\prime}$ principal eigenvectors of $\mathbf{R}$ and spans what is referred to as the signal subspace, while $\mathbf{E}_{n}$ containing the remaining $m-d^{\prime}$ eigenvectors is spanning the noise subspace. In addition, $\boldsymbol{\Lambda}_{s}$ contains the associated signal eigenvalues, and $\boldsymbol{\Lambda}_{n}=$ $\sigma^{2} \mathbf{I}_{m-d^{\prime}}$. It can be shown that $\mathbf{E}_{s}=\mathbf{A}_{0} \mathbf{T}$, where $\mathbf{T} \in \mathbb{C}^{d \times d^{\prime}}$ is a full-rank matrix. Based on this fact we introduce a matrix $\mathbf{B}(\boldsymbol{\theta})$ which spans the null space of $\mathbf{A}(\boldsymbol{\theta})$; thus $\mathbf{B}^{*}(\boldsymbol{\theta}) \mathbf{A}(\boldsymbol{\theta})=\mathbf{0}$ which immediately gives $\mathbf{B}^{*}\left(\boldsymbol{\theta}_{0}\right) \mathbf{E}_{s}=\mathbf{0}$. In the case of a ULA at the receiving antenna, we can write

$$
\mathbf{B}^{*}=\left[\begin{array}{ccccccc}
b_{d} & \ldots & b_{1} & b_{0} & 0 & \ldots & 0 \\
0 & b_{d} & \ldots & b_{1} & b_{0} & \ddots & \vdots \\
\vdots & \ddots & \ddots & & \ddots & \ddots & 0 \\
0 & \ldots & 0 & b_{d} & \ldots & b_{1} & b_{0}
\end{array}\right]
$$

where the coefficients $b_{i}$ are defined by the polynomial having its roots at the DOAs $\boldsymbol{\theta}$ according to

$$
b_{0} z^{d}+b_{1} z^{d-1}+\ldots+b_{d}=b_{0} \prod_{i=1}^{d}\left(z-e^{j \phi_{i}}\right),
$$

with $\phi_{i}$ defined in (3).

However, $\mathbf{E}_{s}$ is not immediately accessible to us, and must be estimated from data. This is preferably done using

$$
\hat{\mathbf{R}}=\frac{1}{N} \sum_{t=1}^{N} \mathbf{x}(t) \mathbf{x}^{*}(t)
$$

and then, similarly to (8),

$$
\hat{\mathbf{R}}=\hat{\mathbf{E}}_{s} \hat{\mathbf{\Lambda}}_{s} \hat{\mathbf{E}}_{s}^{*}+\hat{\mathbf{E}}_{n} \hat{\mathbf{\Lambda}}_{n} \hat{\mathbf{E}}_{n}^{*} .
$$

We can thus estimate $\boldsymbol{\theta}$ by minimizing a suitable norm of the residual

$$
\boldsymbol{\epsilon}=\operatorname{vec}\left(\mathbf{B}^{*}(\boldsymbol{\theta}) \hat{\mathbf{E}}_{s}\right),
$$

where $\operatorname{vec}(\cdot)$ is the vecorization operator which stacks the columns of a matrix on top of each other. Due to finite sample effects, (13) will in general not be equal to zero even for the true parameter values. In order to correctly minimize this residual, we must ensure that both the real and the imaginary parts are minimized. To that end and following [6], we introduce the notation

$$
\begin{aligned}
\hat{\mathbf{e}}_{s} & =\left[\begin{array}{l}
\operatorname{vec}\left(\hat{\mathbf{E}}_{s}\right) \\
\operatorname{vec}\left(\hat{\mathbf{E}}_{s}^{\mathrm{c}}\right)
\end{array}\right] \\
\underline{\mathbf{A}} & =\left[\begin{array}{cc}
\left(\mathbf{I}_{d^{\prime}} \otimes \mathbf{A}\right) & \mathbf{0} \\
\mathbf{0} & \left(\mathbf{I}_{d^{\prime}} \otimes \mathbf{A}^{\mathrm{c}}\right)
\end{array}\right] \\
\underline{\mathbf{B}} & =\left[\begin{array}{cc}
\left(\mathbf{I}_{d^{\prime}} \otimes \mathbf{B}\right) & \mathbf{0} \\
\mathbf{0} & \left(\mathbf{I}_{d^{\prime}} \otimes \mathbf{B}^{\mathrm{c}}\right)
\end{array}\right] \\
\underline{\boldsymbol{\epsilon}} & =\left[\begin{array}{c}
\boldsymbol{\epsilon} \\
\boldsymbol{\epsilon}^{\mathrm{c}}
\end{array}\right]=\underline{\mathbf{B}}^{*} \hat{\mathbf{e}}_{s},
\end{aligned}
$$

where the superscript ${ }^{\mathrm{c}}$ denotes complex conjugate, and $\otimes$ denotes the Kronecker product. With this definition of $\underline{\epsilon}$ we can formulate a minimization criteria that can be used to estimate the DOAs:

$$
\begin{aligned}
\hat{\boldsymbol{\theta}} & =\underset{\boldsymbol{\theta}}{\arg \min } V(\boldsymbol{\theta}), \\
V(\boldsymbol{\theta}) & =\underline{\boldsymbol{\epsilon}}^{*}(\boldsymbol{\theta}) \mathbf{W} \underline{\boldsymbol{\epsilon}}(\boldsymbol{\theta}) .
\end{aligned}
$$

In the expression (18), $\mathbf{W}$ refers to a positive definite weighting matrix chosen such that the asymptotic variance of the estimates $\hat{\boldsymbol{\theta}}$ are minimized. Note that the optimal choice realizes the asymptotically statistically efficient algorithms in [7] and [8].

With (9) we can rewrite (13) according to

$$
\begin{aligned}
\boldsymbol{\epsilon} & =\operatorname{vec}\left(\mathbf{B}^{*}(\boldsymbol{\theta}) \hat{\mathbf{E}}_{s}\right)=\left(\hat{\mathbf{E}}_{s}^{\mathrm{T}} \otimes \mathbf{I}_{m-d}\right) \operatorname{vec}\left(\mathbf{B}^{*}\right) \\
& =\left(\hat{\mathbf{E}}_{s}^{\mathrm{T}} \otimes \mathbf{I}_{m-d}\right) \mathbf{\Phi}_{\mathbf{B b}} \mathbf{b} \triangleq \mathbf{Q b},
\end{aligned}
$$

where we have utilized that vec $\left(\mathbf{A}_{1} \mathbf{A}_{2} \mathbf{A}_{3}\right)=\left(\mathbf{A}_{3}^{\mathrm{T}} \otimes \mathbf{A}_{1}\right)$ vec $\mathbf{A}_{2}$ for arbitrary matrices of suitable dimensions, introduced $\boldsymbol{\Phi}_{\mathrm{Bb}}$ as the selection matrix mapping the elements of $\mathbf{b}=\left[\begin{array}{llll}b_{0} & b_{1} & \cdots & b_{d}\end{array}\right]^{\mathrm{T}}$ to $\mathbf{B}^{*}$ and defined $\mathbf{Q} \triangleq\left(\hat{\mathbf{E}}_{s}^{\mathrm{T}} \otimes \mathbf{I}_{m-d}\right) \mathbf{\Phi}_{\mathbf{B b}}$. Using (19) in (18), we get the expression

$$
V(\boldsymbol{\theta})=\underline{\mathbf{b}}^{*} \underline{\mathbf{Q}}^{*} \mathbf{W} \underline{\mathbf{Q}} \underline{\mathbf{b}},
$$

where

$$
\begin{aligned}
& \underline{\mathbf{Q}}=\left[\begin{array}{ll}
\mathbf{Q} & \mathbf{0} \\
\mathbf{0} & \mathbf{Q}^{\mathrm{c}}
\end{array}\right], \\
& \underline{\mathbf{b}}=\left[\begin{array}{c}
\mathbf{b} \\
\mathbf{b}^{\mathrm{c}}
\end{array}\right] .
\end{aligned}
$$

The reason to rewrite in this manner is that the minimization of (18) is greatly simplified when parameterized by $\mathbf{b}$ as in (20); through (10) there is a simple mapping between the elements of $\mathbf{b}$ and the DOAs.

Remark: Note that the roots of (10) are constrained to the unit circle. To enforce that constraint when solving (20) significantly complicates the minimization procedure. We can relax this constraint to $\mathbf{b}$ being conjugate symmetric (i.e., $b_{i}=b_{d-i}^{\mathrm{c}}, i=$ $0, \ldots, d)$ and still obtain good accuracy. This has the advantage that the conjugate symmetry constraint can be algebraically enforced, allowing an unconstrained minimization of a slightly different expression than (20). Also note that to avoid the trivial solution $\mathbf{b}=\mathbf{0}$ a norm constraint should be enforced, i.e. $\|\mathbf{b}\|=1$ where $\|\cdot\|$ is the euclidian norm, or by constraining a specific element of $\mathbf{b}$, e.g. $\operatorname{Re}\left(b_{0}\right)=1$. Such constraints can also be enforced without complicating the minimization step. See, e.g., [6] for more and exact details on this minimization. 


\subsection{Exact prior knowledge}

When some of the DOAs $\boldsymbol{\theta}_{k}$ are known exactly these known roots can be factored from the polynomial (10) according to the PLEDGE framework [2]

$$
b_{0} \prod_{i=1}^{d}\left(z-e^{j \phi_{i}}\right)=P_{k}\left(\boldsymbol{\theta}_{k}\right) P_{u}\left(\boldsymbol{\theta}_{u}\right) .
$$

Let the coefficients of the known polynomial $P_{k}$ constitute the set $\left\{c_{i}\right\}_{i=0}^{d_{k}}$, and use them to form the matrix $\mathbf{C} \in \mathbb{C}^{(d+1) \times\left(d_{u}+1\right)}$ similarly to (9), (10):

$$
\mathbf{C}^{T}=\left[\begin{array}{cccccc}
c_{0} & c_{1} & \ldots & c_{d_{k}} & & \mathbf{0} \\
& \ddots & \ddots & & \ddots & \\
\mathbf{0} & & c_{0} & c_{1} & \ldots & c_{d_{k}}
\end{array}\right]
$$

We collect the coefficients of the unknown polynomial in $\tilde{\mathbf{b}}=$ $\left[\begin{array}{llll}\tilde{b}_{0} & \tilde{b}_{1} & \cdots & \tilde{b}_{d_{u}}\end{array}\right]^{\mathrm{T}}$. We can then write the relation (23) in matrix notation as

$$
\mathbf{b}=\mathbf{C} \tilde{\mathbf{b}}
$$

With (25) it is immediate to modify (22) and (20) to take the known sources into account, viz.:

$$
V\left(\boldsymbol{\theta}_{u}\right)=\underline{\tilde{\mathbf{b}}}^{*} \underline{\mathbf{Q}}_{2}^{*} \mathbf{W} \underline{\mathbf{Q}}_{2} \underline{\tilde{\mathbf{b}}},
$$

where

$$
\begin{aligned}
\underline{\tilde{\mathbf{Q}}} & =\left[\begin{array}{cc}
\mathbf{Q C} & \mathbf{0} \\
\mathbf{0} & \mathbf{Q}^{\mathrm{c}} \mathbf{C}^{\mathrm{c}}
\end{array}\right] \\
\underline{\tilde{\mathbf{b}}} & =\left[\begin{array}{c}
\tilde{\mathbf{b}} \\
\tilde{\mathbf{b}}^{\mathrm{c}}
\end{array}\right] .
\end{aligned}
$$

\subsection{Uncertainty in prior knowledge}

We now turn to the scenario of primary interest to this article, namely the one in which we know some target locations with a given uncertainty. This translates into there being some uncertainty in the C-matrix.

In [6], a scenario with a criterion function as given by (26) is analyzed in the context of there being some uncertainty in the $\mathbf{A}$ matrix; more exactly, some of the controlling array parameters are allowed to be random according to $\rho \sim \mathcal{N}\left(\rho_{0}, \boldsymbol{\Omega}\right)$. In the context of the scenario studied in this article, this translates into there being uncertainty in the elements of the columns of $\mathbf{A}(\boldsymbol{\theta})$ representing the known angles. Hence, we can exploit the solution proposed to the problem given in [6] in order to correctly account for the uncertainty in the known DOAs.

Thus, in order to minimize the criterion function (26), we follow [6] and choose $\mathbf{W}=\mathbf{C}_{\underline{\epsilon}}^{-1}$, where

$$
\mathbf{C}_{\underline{\epsilon}}=\lim _{N \rightarrow \infty} N \mathrm{E}\left[\underline{\epsilon}^{*}\right]
$$

which can be written

$$
\mathbf{C}_{\underline{\epsilon}}=\underline{\mathbf{L}}+\underline{\mathbf{G G}}^{*}
$$

where

$$
\begin{array}{ll}
\underline{\mathbf{L}}=\left[\begin{array}{cc}
\mathbf{L} & \mathbf{0} \\
\mathbf{0} & \mathbf{L}^{\mathrm{c}}
\end{array}\right], & \mathbf{L}=\left(\sigma^{2} \tilde{\boldsymbol{\Lambda}}^{-2} \boldsymbol{\Lambda}_{s} \otimes \mathbf{B}^{*} \mathbf{B}\right), \\
\underline{\mathbf{G}}=\left[\begin{array}{c}
\mathbf{G} \\
\mathbf{G}^{\mathrm{c}}
\end{array}\right], & \mathbf{G}=\left(\mathbf{T}^{\mathrm{T}} \otimes \mathbf{B}^{*}\right) \overline{\mathbf{D}}_{k} \overline{\mathbf{\Theta}}^{1 / 2} .
\end{array}
$$

Here $\tilde{\boldsymbol{\Lambda}}=\boldsymbol{\Lambda}_{s}-\sigma^{2} \mathbf{I}_{d^{\prime}}, \mathbf{T}=\mathbf{A}_{0}^{\dagger} \mathbf{E}_{s}$, where ${ }^{\dagger}$ denotes the MoorePenrose Pseudo-inverse, $\overline{\boldsymbol{\Theta}}^{1 / 2}$ is a symmetric square root of $(N \cdot \boldsymbol{\Theta})$, and $\overline{\mathbf{D}}_{k}$ is defined as

$$
\overline{\mathbf{D}}_{k}=\left[\begin{array}{lll}
\frac{\partial \operatorname{vec}(\mathbf{A}(\boldsymbol{\theta}))}{\partial \theta_{k_{1}}} & \cdots & \frac{\partial \operatorname{vec}(\mathbf{A}(\boldsymbol{\theta}))}{\partial \theta_{k_{d_{k}}}}
\end{array}\right]
$$

evaluated at $\overline{\boldsymbol{\theta}}_{k}$.

It is additionally shown in [6] that the estimates $\hat{\boldsymbol{\theta}}_{u}$ given by the minimization of (26) with $\mathbf{W}$ chosen according to (30) are asymptotically statistically efficient when using estimated quantities in the expressions (31) and (32).

\subsection{Estimator Implementation}

We now succinctly state the steps of the proposed estimator following the analysis in Section 3.

The method of finding the unknown DOAs is performed according to the following sequence:

1. Form $\mathbf{C}$ and $\overline{\mathbf{D}}_{k}$ using the nominally known positions $\overline{\boldsymbol{\theta}}_{k}$.

2. Perform an eigendecomposition of $\hat{\mathbf{R}},(12)$, to find $\hat{\mathbf{E}}_{s}$ and $\hat{\boldsymbol{\Lambda}}_{s}$; use $\hat{\mathbf{E}}_{s}$ to form $\mathbf{Q}\left(\boldsymbol{\Phi}_{\mathrm{Bb}}\right.$ can be formed offline), and create $\underline{\mathbf{Q}}$ according to (27).

3. Estimate $\hat{\sigma}^{2}=\frac{1}{m-d^{\prime}}\left(\operatorname{Tr}(\hat{\mathbf{R}})-\operatorname{Tr}\left(\hat{\boldsymbol{\Lambda}}_{s}\right)\right)$, and $\hat{\tilde{\boldsymbol{\Lambda}}}=\hat{\boldsymbol{\Lambda}}_{s}-\hat{\sigma}^{2} \mathbf{I}$ to create an estimate of $\mathbf{L}$ in (31).

4. Use $\left(\hat{\mathbf{B}}^{*} \hat{\mathbf{B}}\right)^{-1}=\mathbf{I}$ and $\hat{\mathbf{G}}=\mathbf{0}$ as initial estimates, form $\hat{\mathbf{W}}=\hat{\mathbf{C}}_{\underline{\epsilon}}^{-1}$ according to (30); then minimize (26) to find $\hat{\tilde{\mathbf{b}}}_{\text {INIT }}$, an initial estimate of $\tilde{\mathbf{b}}$.

5. Use $\hat{\tilde{\mathbf{b}}}_{\text {INIT }}$ with (25) to form $\left(\hat{\mathbf{B}}^{*} \hat{\mathbf{B}}\right)^{-1}$ and, additionally, with (10) and (3) to form $\hat{\mathbf{T}}=\mathbf{A}^{\dagger}(\hat{\boldsymbol{\theta}}) \hat{\mathbf{E}}_{s}$. This gives improved estimates of $\hat{\mathbf{L}}$ and $\hat{\mathbf{G}}$, which gives a more accurate $\hat{\mathbf{W}}$.

6. Minimize (26) once again to find $\hat{\tilde{\mathbf{b}}}$, then $\phi_{i}, i=1, \ldots, d_{u}$, by rooting (10), and finally the sought DOAs through (3).

The minimization of the function (26) should be constrained such that $\mathbf{b}$ is conjugate symmetric and such that $\|\mathbf{b}\|=1$. Please see e.g. [6] for suitable ways of enforcing these constraints with the given criterion function.

\section{CRAMÉR-RAO BOUND}

From [6] we have that the estimates $\hat{\boldsymbol{\theta}}_{u}$ given by the method summarized in Section 3.3 are asymptotically unbiased and that their asymptotic variance is equal to the Cramér-Rao Bound (CRB). We now desire to find that bound.

Since we are only concerned with the DOA estimation we note from, e.g., [8] that the bound for the estimation of a deterministic ${ }^{1} \boldsymbol{\theta}$ is given by

$$
\mathbf{C}_{\boldsymbol{\theta}}=\frac{\sigma^{2}}{2 N} \operatorname{Re}\left(\left(\mathbf{D}^{*} \boldsymbol{\Pi}_{\mathbf{A}}^{\perp} \mathbf{D}\right) \odot\left(\mathbf{P}^{*} \mathbf{A}^{*} \mathbf{R}^{-1} \mathbf{A} \mathbf{P}\right)^{\mathrm{T}}\right)^{-1}
$$

where

$$
\mathbf{D}=\left[\begin{array}{lll}
\frac{\partial \mathbf{a}\left(\theta_{1}\right)}{\partial \theta_{1}} & \cdots & \frac{\partial \mathbf{a}\left(\theta_{d}\right)}{\partial \theta_{d}}
\end{array}\right],
$$

and $\mathbf{\Pi}_{\mathbf{A}}^{\perp}=\mathbf{I}_{m}-\mathbf{A}\left(\mathbf{A}^{*} \mathbf{A}\right)^{-1} \mathbf{A}^{*}$. Without loss of generality, we partition $\boldsymbol{\theta}=\left[\begin{array}{ll}\overline{\boldsymbol{\theta}}_{u}^{\mathrm{T}} & \overline{\boldsymbol{\theta}}_{k}^{\mathrm{T}}\end{array}\right]^{\mathrm{T}}$, simultaneously partitioning (34) into a

\footnotetext{
${ }^{1}$ Not to be confused with the deterministic CRB
} 
block matrix. Following [4], [5] we can, under the assumption that $\boldsymbol{\theta}_{k}$ is close to $\overline{\boldsymbol{\theta}}_{k}$, modify the lower right block of $\mathbf{C}_{\boldsymbol{\theta}}$ to take the stochastic nature of $\boldsymbol{\theta}_{k}$ into account; doing so we arrive at

$$
\tilde{\mathbf{C}}_{\boldsymbol{\theta}}=\frac{\sigma^{2}}{2 N}\left[\begin{array}{ll}
\mathbf{C}_{\mathrm{P}} & \mathbf{F} \\
\mathbf{F}^{\mathrm{T}} & \boldsymbol{\Gamma}
\end{array}\right]^{-1},
$$

in which

$$
\begin{aligned}
\mathbf{C}_{\mathbf{P}} & =\operatorname{Re}\left(\left(\mathbf{D}_{u}^{*} \boldsymbol{\Pi}_{\mathbf{A}}^{\perp} \mathbf{D}_{u}\right) \odot\left(\overline{\mathbf{P}}_{u}^{*} \mathbf{A}^{*} \mathbf{R}^{-1} \mathbf{A} \overline{\mathbf{P}}_{u}\right)^{\mathrm{T}}\right) \\
\mathbf{F} & =\operatorname{Re}\left(\left(\mathbf{D}_{u}^{*} \boldsymbol{\Pi}_{\mathbf{A}}^{\perp} \mathbf{D}_{k}\right) \odot\left(\overline{\mathbf{P}}_{k}^{*} \mathbf{A}^{*} \mathbf{R}^{-1} \mathbf{A} \overline{\mathbf{P}}_{u}\right)^{\mathrm{T}}\right) \\
\boldsymbol{\Gamma} & =\operatorname{Re}\left(\left(\mathbf{D}_{k}^{*} \boldsymbol{\Pi}_{\mathbf{A}}^{\perp} \mathbf{D}_{k}\right) \odot\left(\overline{\mathbf{P}}_{k}^{*} \mathbf{A}^{*} \mathbf{R}^{-1} \mathbf{A} \overline{\mathbf{P}}_{k}\right)^{\mathrm{T}}\right)+\frac{\sigma^{2}}{2 N} \mathbf{\Theta}^{-1}
\end{aligned}
$$

In these equations, the subscript $u$ and $k$ on $\mathbf{D}$ signifies that the derivatives in (35) are only with respect to the unknown and known sources, respectively, while $\overline{\mathbf{P}}_{u}$ denotes the sub-matrix of $\mathbf{P}$ containing the columns associated with the unknown sources, and $\overline{\mathbf{P}}_{k}$ defined analogously. Thus, using a standard result on block-matrix inversion, the CRB for the unknown DOAs is found as

$$
\operatorname{CRB}\left(\boldsymbol{\theta}_{u}\right)=\frac{\sigma^{2}}{2 N}\left(\mathbf{C}_{\mathrm{P}}-\mathbf{F} \boldsymbol{\Gamma}^{-1} \mathbf{F}^{\mathrm{T}}\right)^{-1} .
$$

We can also note that $\frac{\sigma^{2}}{2 N} \mathbf{C}_{\mathrm{P}}^{-1}$ is the CRB of the estimates $\hat{\boldsymbol{\theta}}_{u}$ when there is no uncertainty in the prior; see [2].

\section{SIMULATIONS}

To study the empirical performance offered by the method in Section 3, we perform numerical Monte Carlo simulations. We simulate a scenario with two sources; the unknown is located at $\bar{\theta}_{u}=15^{\circ}$ and the known source is, for each Monte Carlo simulation, a realization of the random variable $\theta_{k} \sim \mathcal{N}\left(\bar{\theta}_{k}, \Theta\right)$ with $\bar{\theta}_{k}=12^{\circ}$ and $\Theta=0.2^{2}\left[\mathrm{deg}^{2}\right]$. The sources are perfectly correlated which gives $\mathbf{P}=10^{\mathrm{SNR} / 10}\left[\begin{array}{ll}1 & \rho \\ \rho^{\mathrm{c}} & 1\end{array}\right]$. We choose the correlation phase $\rho=\exp (-j 0.72)$ in order to create a difficult estimation scenario.

We compare the accuracy of three estimators in terms of the root-mean square error (RMSE) along with the respective CRB: MODE/WSF [7], [8] which is optimal in the case of no prior information; PLEDGE [2] which is optimal in the case of exact prior information; and the method proposed in this paper, hereby termed R-PLEDGE ("Robust PLEDGE"). The assumptions for the respective CRBs are: no prior information (denoted "CRB ${ }_{\text {SML") }}$ [8]; exact prior information ("CRB ${ }_{P}$ ") [2], (37); and stochastic prior information ("CRB $\mathrm{SP}$ "), (40).

As can be seen in Fig. 1 the proposed method R-PLEDGE and PLEDGE are about as accurate when the noise induced estimation error is larger than the variance of the known source. However, as the SNR increases the known source variance becomes predominant and PLEDGE is not able to benefit from the higher SNR due to the error in the prior. R-PLEDGE is seen to transition to the accuracy of the no-prior estimator, MODE, in this case, and can thus deliver as accurate estimates as is possible. For very high SNRs, however, R-PLEDGE begins to suffer from the error in the prior, and it is seen that its estimates are worse than those of MODE. However, the feasible region in which the prior information is usable has been significantly enhanced; a slightly higher threshold seems to be the only negative effects of R-PLEDGE as compared to PLEDGE. Hence RPLEDGE generalizes PLEDGE to efficiently handle the case of uncertain prior knowledge.

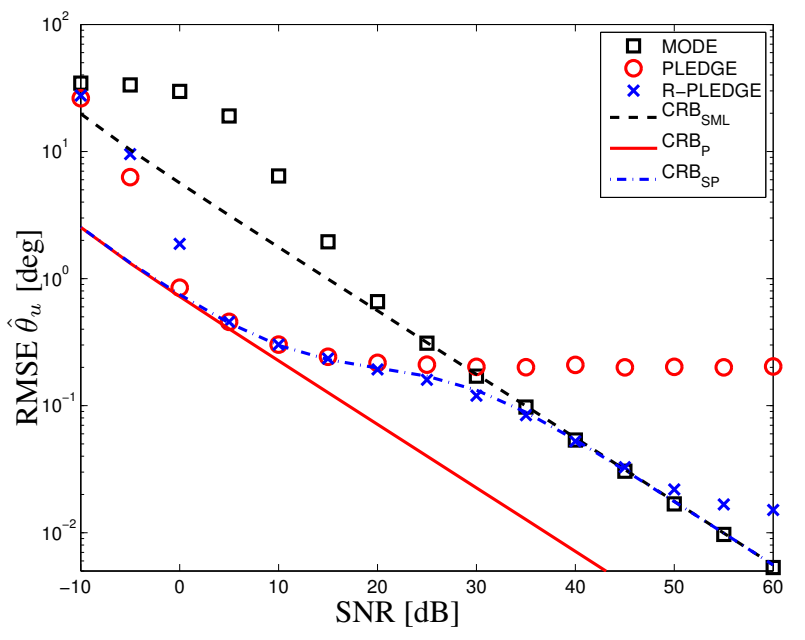

Fig. 1. RMSE of 3 estimators and CRBs, averages of $4000 \mathrm{MC}$ realizations; $N=100, \bar{\theta}_{u}=15^{\circ}, \bar{\theta}_{k}=12^{\circ}, \Theta=0.2^{2}, m=10$.

\section{CONCLUSIONS}

In this paper we have developed a DOA estimator that can exploit prior information on some DOAs, even if the uncertainty in this knowledge is of the same order of magnitude as the expected unaided estimation accuracy. We have also developed an expression for the $\mathrm{CRB}$ of the problem under consideration, and this bound was seen to be attainable at moderate SNRs.

\section{REFERENCES}

[1] R.D. DeGroat, E.M. Dowling, and D.A. Linebarger, "The constrained MUSIC problem," IEEE Trans. Signal Process., vol. 41, no. 3, pp. 1445-1449, Mar. 1993.

[2] G. Bouleux, P. Stoica, and R. Boyer, "An optimal prior knowledge-based DOA estimation method," in 17th European Signal Process. Conf., Glasgow, UK, Aug. 2009, pp. 869-873.

[3] P. Wirfält, M. Jansson, G. Bouleux, and P. Stoica, "Prior knowledge-based direction of arrival estimation," in IEEE Int. Conf. on Acoust., Speech and Signal Process., Prague, Czech Republic, May 2011, pp. 2540-2543.

[4] B. Wahlberg, B. Ottersten, and M. Viberg, "Robust signal parameter estimation in the presence of array perturbations," in IEEE Int. Conf. on Acoust., Speech and Signal Process., Toronto, Canada, Apr. 1991, pp. 3277-3280 vol.5.

[5] M. Viberg and A.L. Swindlehurst, "A bayesian approach to auto-calibration for parametric array signal processing," IEEE Trans. on Signal Process., vol. 42, no. 12, pp. 3495-3507, Dec. 1994.

[6] M. Jansson, A.L. Swindlehurst, and B. Ottersten, "Weighted subspace fitting for general array error models," IEEE Trans. on Signal Process., vol. 46, no. 9, pp. 2484-2498, Sep. 1998.

[7] P. Stoica and K. Sharman, "Maximum likelihood methods for direction-of-arrival estimation," IEEE Trans. Acoust., Speech, Signal Process., vol. 38, no. 7, pp. 1132-1143, Jul. 1990.

[8] M. Viberg and B. Ottersten, "Sensor array processing based on subspace fitting," IEEE Trans. on Signal Process., vol. 39, no. 5, pp. 1110-1121, May 1991. 